\title{
Transporte longitudinal de sedimentos no litoral da planície deltaica do Rio Doce
}

\section{Littoral longshore sediment transport along the deltaic plain of Rio Doce}

\section{${ }^{\text {ac }}$ Tiago Castro Alves Oliveira, ${ }^{\text {bd }}$ Jacqueline Albino, ${ }^{\text {be }}$ Igor Venancio}

\author{
${ }^{a}$ Programa de Pós-graduação de Engenharia Ambiental, Universidade Federal do Espírito Santo, ${ }^{b}$ Departamento de Oceanografia e \\ Ecologia, Universidade Federal do Espírito Santo
}

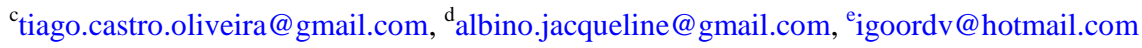

\begin{abstract}
Resumo
Neste trabalho estuda-se o potencial de transporte longitudinal de sedimento por ação de ondas no litoral da planície deltaica do Rio Doce. Considerando o clima de ondas entre 1948 e 2008 calcula-se o sentido do transporte longitudinal de sedimentos predominantes ao longo do ano, bem como a sua variabilidade sazonal e os volumes de sedimentos transportados. Os resultados obtidos indicam que o transporte longitudinal pela ação das ondas ocorre essencialmente de Sul para Norte. No Inverno e no Outono o transporte de sedimentos pela deriva litorânea é maior. A magnitude do transporte para Sul é maior a Sul da desembocadura do Rio Doce, mas não consegue alterar o sentido do transporte líquido que é para norte ao longo de toda a área estudada. Os resultados obtidos mostram que os sedimentos aportados pelo rio Doce são transportados preferencialmente para norte devido à ação das ondas, o que corrobora esquemas de evolução paleo-geográfica publicados anteriormente.
\end{abstract}

Palavras-chave: Transporte litorâneo de sedimentos; Planície deltaica do Rio Doce; Sistema de Modelado Costeiro do Brasil

\begin{abstract}
This work is focused on the study of the longshore littoral sediment transport induced by waves in the deltaic plain of Rio Doce. Based on the wave climate from 1948 to 2008 the annual longshore sediment transport directions and volumes and its seasonality are calculated. Obtained results show that the longshore sediment transport induced by waves is mainly from South to North. During winter and autumn the sediment transport reaches the maximum rates. Sediment transport to the south is greater south of the Rio Doce mouth, although it not able to revert the direction of the net longshore transport, witch is northwards along the entire study area. The observed sediment transport pattern is in agreement with the coastal evolution models available for the region and agrees with the paleogeographic evolution schemes published for the study area.
\end{abstract}

Key-words: Littoral sediments transport; Rio Doce deltaic plain; SMC-Brasil

\section{Introdução}

A planície deltaica do rio Doce, com largura máxima de $40 \mathrm{~km}$ e extensão em torno de $160 \mathrm{~km}$, corresponde ao maior registro de depósitos sedimentares quaternários do Estado do Espírito Santo. Trata-se de um delta dominado por ondas onde as evidências morfoestratigráficas destacam a atuação da deriva litorânea de sedimentos, da carga e descarga fluvial e das variações do nível do mar na sua evolução (Dominguez et al. 1981, Dominguez et al. 1983, Martin et al. 1996, Martin et al. 1997).

Reconstituições paleo-geográficas das cristas de praia durante a progradação Holocênica sugerem discordâncias nos alinhamentos destas cristas, decorrentes de episódios erosivos associado a inversões no sentido da deriva litorânea. Atualmente o sentido preferencial da deriva é de sul para norte. Os sedimentos fluviais são transportados, predominantemente, nesta direção (Martin et al. 2003), resultando em uma progradação mais acentuada da planície costeira a norte da desembocadura.

Ao sul da desembocadura, a orientação geral da linha de costa voltada para sudeste, favorece também a deposição dos sedimentos fluviais. Considera-se ainda que, os sedimentos trazidos pela deriva são trapeados pelo efeito do molhe hidráulico produzido pelo efluente fluvial (Dominguez et al. 1983).

Mudanças na frequência direcional das ondas incidentes ao longo da costa durante a evolução do rio Doce foi constatada pela modelagem numérica realizada por Bittencourt et al. (2007). Os autores adotando o padrão de ventos atual e o processo de refração das ondas junto à linha de costa, concluíram que a dispersão dos sedimentos potencial é concordante com a evolução descrita pelas interpretações morfoestratigraficas. Os autores ainda sugeriram que, em média, as condições de contorno atuais parecem ter 
permanecido com as mesmas características gerais desde o Holoceno Tardio até hoje.

Estudos de perfis praiais adjacentes à desembocadura do rio Doce mostram que após eventos de alta descarga, ocorre progradação dos perfis das praias tanto a norte quanto a sul da desembocadura (Albino \& Suguio 2010). Sob condições hidrológicas do rio próximas às condições médias, as alterações sazonais na frequência direcional das ondas resultam alternadamente em progradação e/ou recuo dos perfis ora ao sul ora ao norte da desembocadura (Albino \& Suguio 2010, Albino et al. 2006).

A morfologia e mobilidade da linha de costa da planície deltaica do rio Doce estão diretamente relacionadas ao transporte longitudinal. Seu padrão determina a geometria da linha de costa e consequentemente sua progradação ou retrogradação em escala sazonal e/ou milenar.

O entendimento da deriva litorânea e do transporte de sedimentos associados é necessário para se compreender a mobilidade da linha de costa na zona adjacente à foz do Rio Doce. A quantificação do transporte de sedimentos torna-se fundamental para a compreensão da evolução da linha de costa (Oliveira et al. 2004, Jimenez \& Sánchez-Arcilla 2004, Khalifa et al. 2009, Silva et al. 2013, Viola et al. 2014). Desta forma, na área de estudo esta quantificação pode contribuir na compreensão deste processo evolutivo.

Neste contexto, o objetivo principal deste trabalho é quantificar o transporte longitudinal de sedimentos por ação das ondas ao longo da planície deltaica do Rio Doce e compreender a sua variabilidade espacial e sazonal.

\section{Metodologia}

A metodologia deste estudo consistiu numa análise do clima de ondas a aproximadamente $24 \mathrm{~m}$ de profundidade, propagação das ondas até à costa e consequente cálculo do potencial de transporte de sedimentos. A realização destes três passos que se descreve em seguida teve como base o uso do Sistema de Modelagem Costeiro do Brasil (SMC-Brasil). O SMC é um aplicativo que incorpora uma serie de modelos numéricos (González et al. 2007, González et al. 2010, Raabe et al. 2010) e banco de dados de cartas náuticas, ondas e nível do mar, os quais permitem implementar a metodologia de estudo e desenho de atuações no litoral brasileiro.

\subsection{Dados de ondas}

Devido à inexistência de dados medidos para a área de estudo recorreu-se a dados de ondas produzidos por modelos numéricos de hindcast. Dados de ondas para o período entre 1948 e 2008, foram obtidos através do modelo Global Ocean Wave (GOW), que consiste numa reanálise de ondas global (Reguero et al. 2012).

As séries temporais dos diferentes parâmetros estatísticos da onda e da energia espectral podem ser obtidas pelo GOW numa malha global com resolução de $1.5^{\circ}$ em longitude e $1.0^{\circ}$ em latitude e para uma resolução temporal horária. O modelo GOW foi calibrado e validado globalmente utilizando medições instrumentais de 21 bóias e por altimetria extraída de imagens satélites (Reguero et al. 2012). O processamento dos dados de altimetria incluiu a identificação e eliminação das alterações associadas às perturbações meteorológicas.

Neste trabalho foram utilizados os resultados fornecidos pelo modelo GOW para o ponto de coordenadas $\quad 19^{\circ} 30^{\prime} 25.20^{\prime \prime} \mathrm{S} \quad 39^{\circ} 36^{\prime} 50.40 " \mathrm{O}$ correspondente a uma profundidade de $24 \mathrm{~m}$. Este ponto situa-se em condições de águas intermediárias em frente à zona de estudo.

\subsection{Propagação de ondas}

A propagação das ondas foi feita com o modelo Oluca-SP (González et al. 2007) que é um modelo espectral não dispersivo que resolve a fase e faz parte do SMC-Brasil.

$\mathrm{O}$ modelo requer como entrada no contorno exterior um estado de mar direcional representado por um espectro bidimensional discretizado nas componentes de frequência e direção, as quais são propagadas de maneira simultânea. O Oluca-SP considera os processos de refração-difração com interação ondacorrente e as perdas de energia devido à quebra das ondas.

A propagação das ondas foi feita utilizando a batimetria das cartas náuticas da Diretoria de Hidrografia e Navegação (DHN) para a zona de estudo. Estas cartas náuticas apresentam escalas variáveis entre 1:12000 e 1:30000 e estão disponíveis no pacote SMC-Brasil.

\subsection{Transporte longitudinal de sedimentos}

O transporte longitudinal de sedimentos foi calculado para 8 perfis na zona de estudo (ver figura 1) usando a fórmula de CERC (1984). Este método baseia-se na hipótese de que o transporte de sedimentos é diretamente proporcional à componente paralela à costa do fluxo de energia na zona de rebentação. Na fórmula de CERC o caudal $Q_{1}$ em $\mathrm{m}^{3} / \mathrm{s}$ de sedimentos transportado por uma onda de altura significativa $\mathrm{H}_{\mathrm{b}}$ no ponto de rebentação é dado por:

$$
Q_{D}=K \cdot H_{b}^{5 / 2} \cdot \operatorname{sen}\left(2 \alpha_{b}\right) \cdot \frac{\rho \sqrt{g}}{16 \gamma^{1 / 2} \cdot\left(\rho_{g}-p\right)(1-n)}
$$

Sendo $\rho_{s}$ a densidade do sedimento, $\rho$ a densidade da água do mar, $\mathbf{n}$ a porosidade do sedimento, $\alpha_{\mathbf{b}}$ o ângulo formado entre as cristas das ondas na rotura em relação à costa, $\gamma$ o parâmetro de critério de quebra, $k$ um coeficiente empírico de proporcionalidade e $g$ a aceleração da gravidade. 

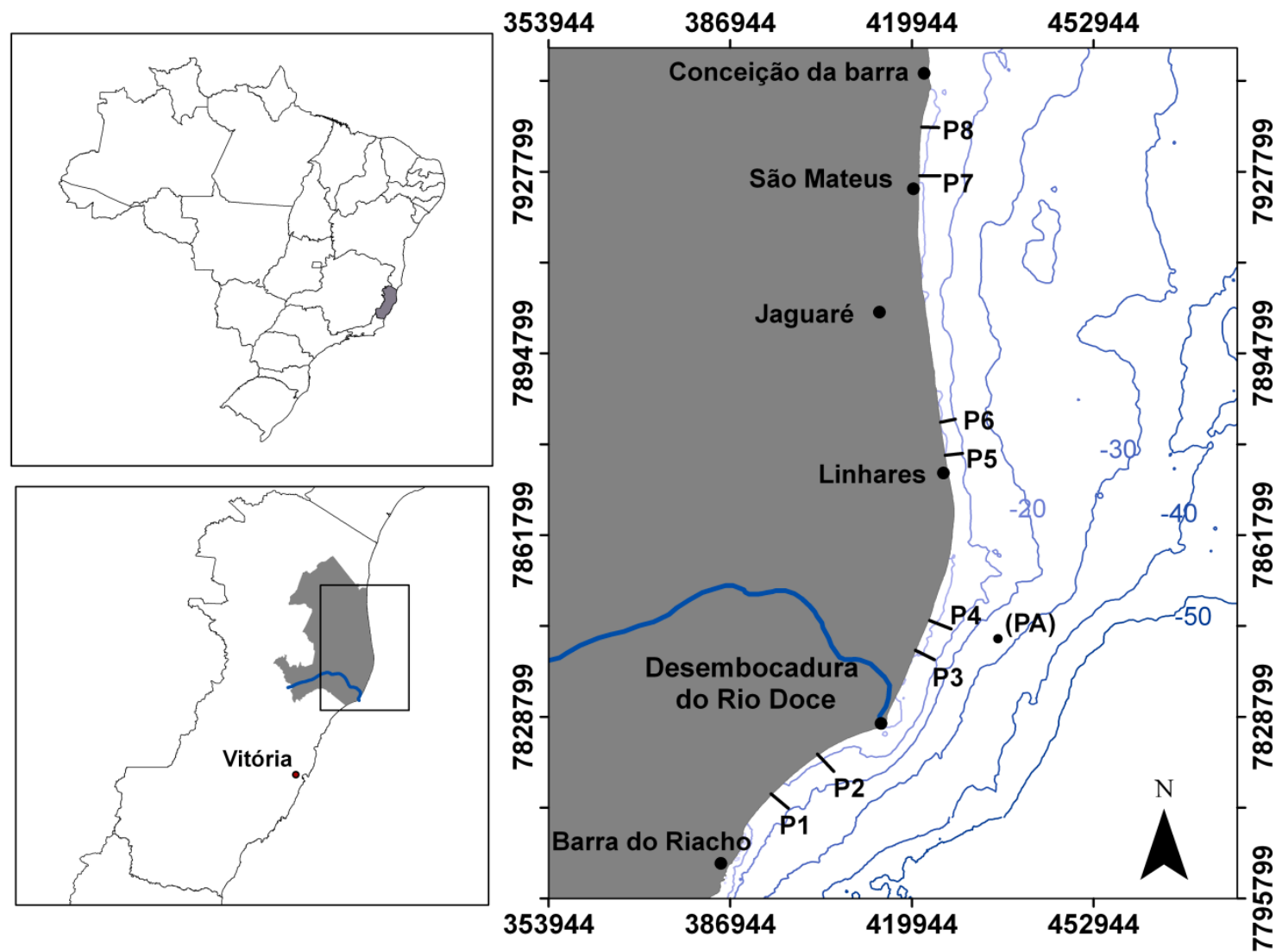

Figura 1: Localização da zona de estudo, do ponto de dados de ondas (PA) e dos perfis onde foi determinado o potencial do transporte longitudinal de sedimentos (P1-P8).

O valor de $K$ foi calculado de acordo com o proposto por Mil-Homens et al. (2013):

$$
K=\left[2232.7\left(\frac{H_{b}}{L_{0}}\right)^{1.45}+4.505\right]^{-1}
$$

onde $L_{0}$ é o comprimento de onda em águas profundas.

\section{Resultados}

A figura 2 mostra a distribuição das alturas de onda significativa $\left(\mathrm{H}_{\mathrm{s}}\right)$ e do período de pico $\left(\mathrm{T}_{\mathrm{p}}\right)$ obtidas para o ponto PA. Verificou-se que $97,3 \%$ das ondas apresentam direção compreendida entre $56,25^{\circ} \mathrm{N}$ e $168,75^{\circ} \mathrm{N}$. O setor Leste é o que registra a maior frequência de ondas, seguido dos setores Leste-sudeste e Sudeste com respectivamente $34,8 \%, 22,1 \%$ e $16,1 \%$ do total de ondas. Em relação às alturas de ondas significativas $89,4 \%$ apresentam valores entre $0,5 \mathrm{~m} \mathrm{e}$ $2,0 \mathrm{~m}$ sendo que $43,9 \%$ encontram-se no intervalo de 1,0 a $1,5 \mathrm{~m}$. Alturas de onda significativas superiores a $2,5 \mathrm{~m}$ representam apenas $2,4 \%$ sendo que a maioria destas ondas $(70 \%)$ pertencem aos setores direcionais Sudeste e Sul-sudeste. A máxima altura significativa nos 60 anos de dados é de 4,2 m.

Em relação aos períodos de pico, $98,3 \%$ dos dados apresentam valores entre 4 e $10 \mathrm{~s}$ sendo que $70,5 \%$ encontram-se no intervalo de 6,0 a $8,0 \mathrm{~s}$. Períodos de pico maiores que $10 \mathrm{~s}$ representam apenas $1,6 \%$ sendo que a maioria desta ondas $(87 \%)$ pertencem aos setores direcionais Sudeste e Sul-sudeste. O máximo período de pico nos 60 anos de dados é de 13,9 s.

A figura 3 mostra os valores calculados para o transporte longitudinal em cada um dos perfis referente a um clima de ondas de 60 anos (1948 a 2008). O transporte líquido é a diferença entre os transportes para Sul e para Norte.

Observa-se que o transporte total varia de perfil para perfil sendo menor no perfil $1\left(47,513 \mathrm{~m}^{3} / \mathrm{ano}\right)$ e maior no perfil $6\left(160,854 \mathrm{~m}^{3} / \mathrm{ano}\right)$. Em todos os perfis o transporte é maior para Norte do que para Sul. Existe uma tendência do transporte líquido e do transporte para Norte aumentar do perfil P1 para P6 e de P8 para P6. Os perfis P5, P6, P7 e P8 apresentam praticamente apenas transporte para Norte sendo nestes casos o transporte para Sul menor que $90 \mathrm{~m}^{3}$ /ano.

Nos perfis P1, P2, P3 e P4 os valores do transporte para Sul é maior que nos demais perfis. Nestes casos o valor do transporte para sul varia entre o mínimo de $5.878 \mathrm{~m}^{3} /$ ano $(\mathrm{P} 4)$ e o máximo de $25.467 \mathrm{~m}^{3} /$ ano $(\mathrm{P} 2)$.

Os resultados do transporte longitudinal de sedimentos anual calculado para o ano de 2008 encontram-se na figura 4. Comparando-se as figuras 4 e 3 pode-se observar um padrão igual na variação espacial (de perfil para perfil) dos valores de transporte, para Norte e para Sul. No entanto verificase que no ano 2008 os valores absolutos para Norte e para Sul foram maiores que no período 1948- 2008. 
O comportamento sazonal do transporte longitudinal de sedimentos no período 1948-2008 é mostrado na figura 5. Durante o Outono e o Inverno os valores absolutos do transporte para Norte são maiores que durante o Verão e a Primavera. O transporte para Sul apresenta valores absolutos aproximadamente uniformes ao longo do ano.

A figura 6 sintetiza o sentido e a magnitude do transporte longitudinal líquido ao longo da linha de costa na planície deltaica do Rio Doce para o período 1948-2008. O transporte líquido anual ao longo de toda a costa é para Norte, com os maiores valores verificados a norte da desembocadura do rio Doce.

\section{Conclusões}

Os resultados obtidos indicam que na área de estudo o transporte longitudinal líquido de sedimentos pela ação das ondas ocorre essencialmente de Sul para Norte. Este transporte varia espacialmente em magnitude entre 26.000 e $120.000 \mathrm{~m}^{3} /$ ano. O volume total de sedimentos transportados anualmente (Sul + Norte) varia espacialmente entre 50.000 e $190.000 \mathrm{~m}^{3} /$ ano.

Durante o Inverno e o Outono é quando ocorrem as maiores taxas de transporte longitudinal de sedimentos. A componente do transporte no sentido Sul apresenta magnitudes maiores a Sul da desembocadura do Rio Doce. Estes valores, entretanto não são suficientes para alterar o sentido do transporte líquido, que embora mais reduzidos, permanece para norte.

Durante o Outono e o Inverno a magnitude do transporte para norte aumenta. A magnitude do transporte para Sul, entretanto apresenta valores baixos e aproximadamente uniformes ao longo do ano. Embora ocorram inversões sazonais no sentido do transporte longitudinal de sedimentos, elas não tem influência nos valores do transporte líquido que ocorre predominante no sentido norte.
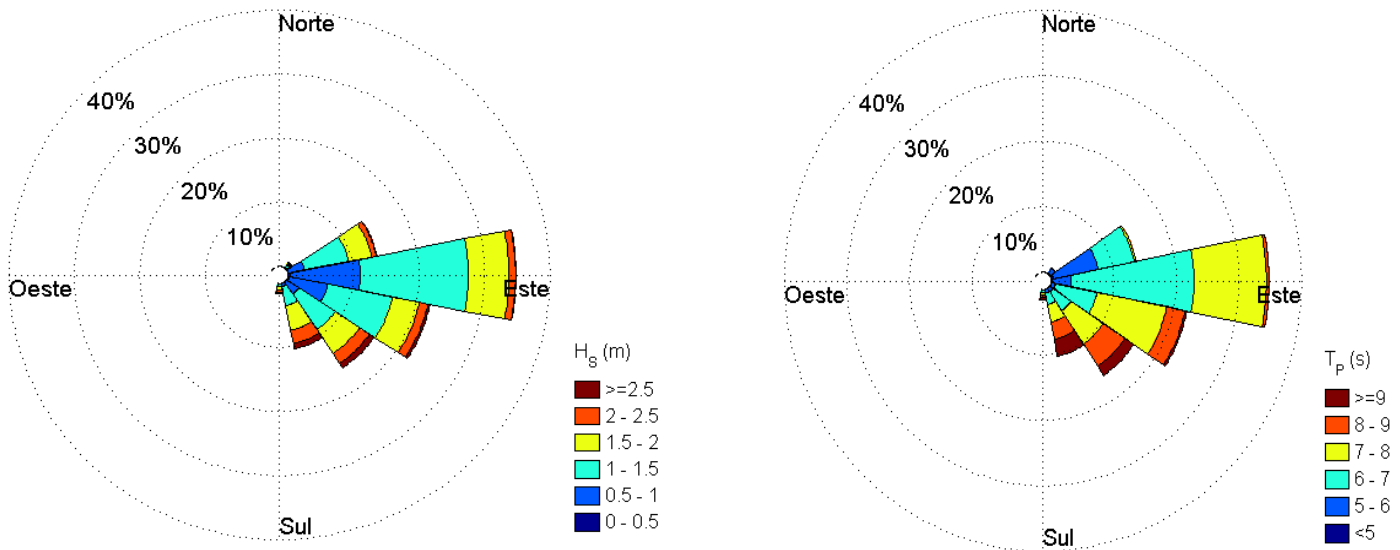

Figura 2: Rosa de altura de onda significativa $\left(\mathrm{H}_{\mathrm{s}}\right)$ e período de pico $\left(\mathrm{T}_{\mathrm{p}}\right)$ em PA.

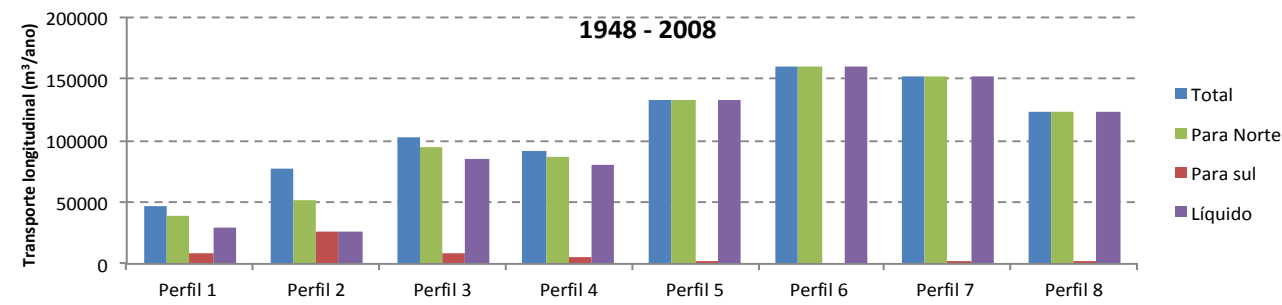

Figura 3: Transporte longitudinal de sedimentos com o clima médio anual calculado com base nos dados de onda entre 1948 e 2008.

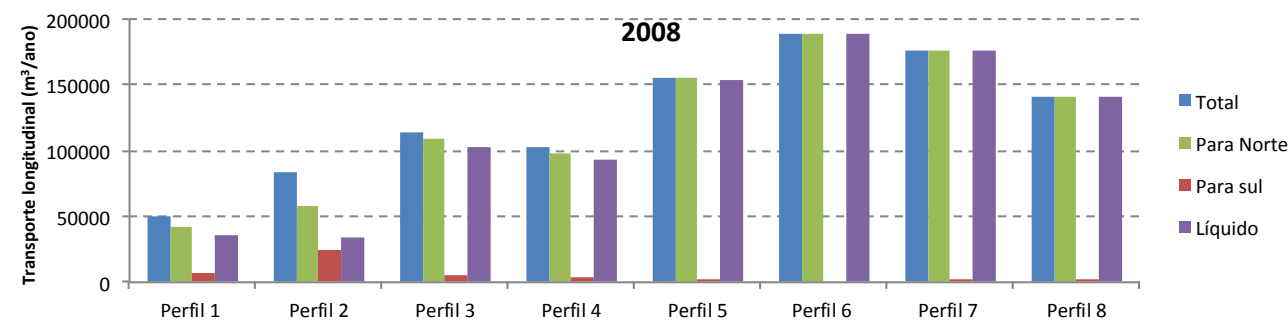

Figura 4: Transporte longitudinal de sedimentos para o ano de 2008. 

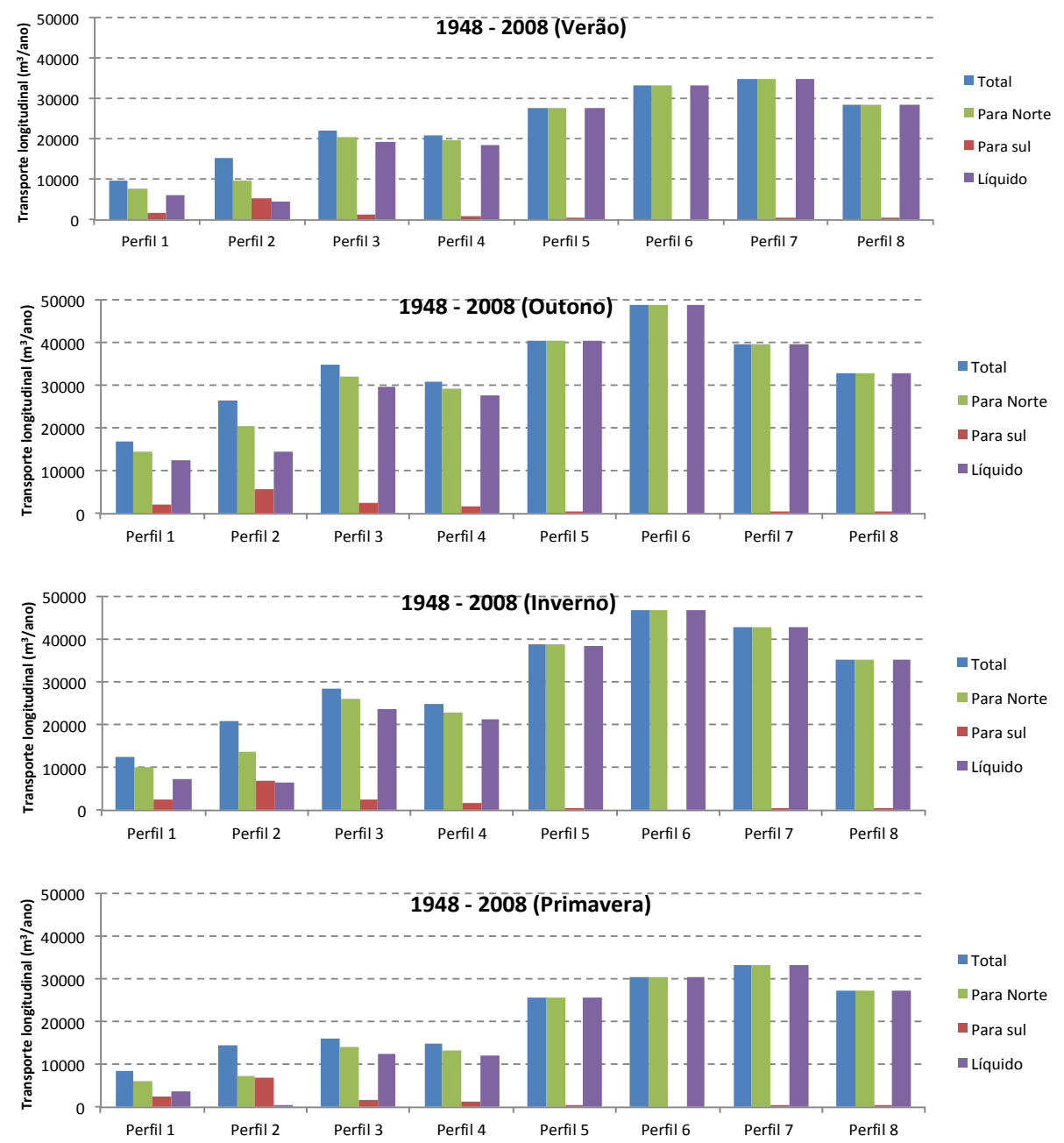

Figura 5: Variação sazonal do transporte longitudinal de sedimentos com o clima médio anual calculado com base nos dados de onda entre 1948 e 2008 .

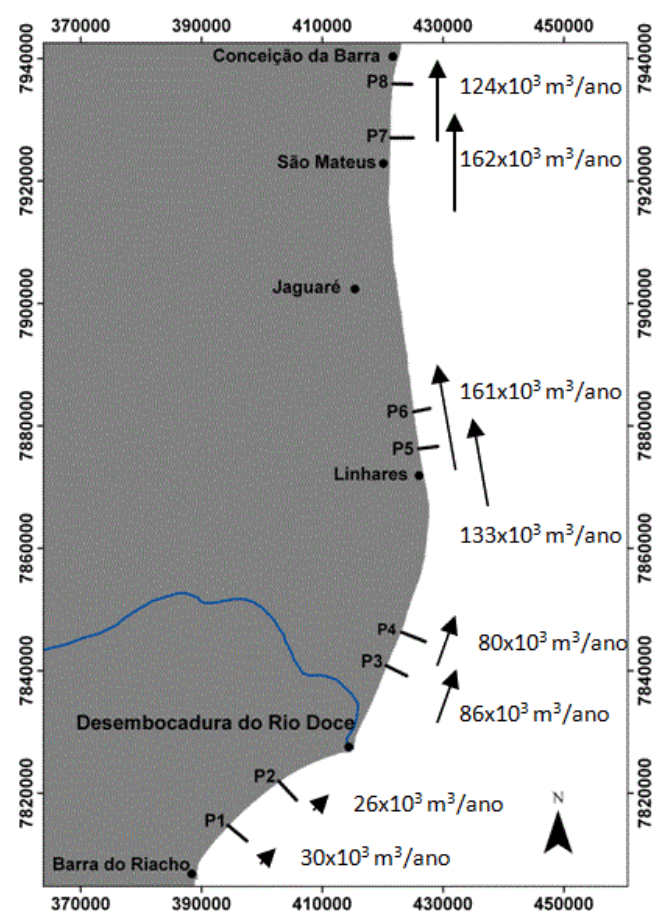

Figura 6: Direção e magnitude do transporte longitudinal líquido de sedimentos no período de 1948-2008
Os resultados mostram que os sedimentos aportados pelo rio Doce são transportados preferencialmente para norte devido à ação das ondas, o que corrobora os esquemas de evolução paleogeográfica publicados anteriormente (Martin et al. 1993).

Os padrões do transporte de sedimentos encontrados a norte da desembocadura do Rio Doce estão também de acordo com os resultados apresentados por Bittencourt et al. (2007). Por outro lado a sul da desembocadura, Bittencourt et al. (2007) obtiveram transporte liquido para sul o que difere dos resultados obtidos neste estudo. Entretanto o transporte líquido, obtido neste estudo, para o trecho a Sul da desembocadura é de pequena magnitude (da ordem dos $26.000 \mathrm{~m}^{3} / \mathrm{ano}$ ) e pequenas variações no clima de ondas podem alterar o sentido do transporte líquido. Desta forma uma futura análise da variabilidade interanual do clima de ondas pode ajudar na identificação de possíveis inversões no sentido do transporte líquido dentro do período investigado.

\section{Agradecimentos}

Os autores agradecem à CAPES o financiamento da bolsa A039_2013 - Atração de Jovens Talentos. Os autores agradecem o suporte ao IH Cantabria, Instituto 
Papel da praia na proteção da costa e as alterações oceanográficas em diferentes escalas temporais

de Hidráulica Ambiental de la Universidad de Cantabria no uso do SMC-Brasil.

\section{Referências Bibliográficas}

Albino J., Suguio K. 2010. Sedimentation processes and beach morphodynamics active at the Doce River mouth, Espírito Santo State. Brazil An. Acad. Bras. Ciênc., 82(4), 1031-1044.

Albino J., Girardi G., Nascimento K.A. 2006. Espirito Santo. In MUEHE, D. Progradação e Erosão do Litoral Brasileiro. Ministério de Meio Ambiente MMA. 227-264.

Bittencourt A. C. S. P., Dominguez J. M., Martin L., Silva I. R., DeMedeiros K. O. 2007. Past and current sediment dispersion pattern estimates through numerical modeling of wave climate: an example of the Holocene delta of the Doce River, Espírito Santo, Brazil. Anais da Academia Brasileira de Ciências, 79 (2), 333-41.

CERC 1984. Shore Protection Manual, fourth ed., US Army Engineer Waterways Experiment Station. US Government Printing Office, Washington, DC.

Dominguez J.M.L., Bittencourt A.C.S.P., Martin L. 1981. Esquema evolutivo da sedimentação quaternária nas feições deltaicas dos rios São Franscisco (SE/AL), Jequitinhonha (BA), Doce (ES) e Paraíba do Sul (RJ). Rev. Brasil. Geoc. 11(4):227-237.

Dominguez J.M.L., Bittencourt A.C.S.P., Martin L. 1983. O papel da deriva litorânea de sedimentos arenosos na construção das planícies costeiras associadas a desembocaduras dos rios São Franscisco (SE/AL), Jequitinhonha (BA), Doce (ES) e Paraíba do Sul (RJ). Rev. Brasil. Geoc.13(4):93-105.

González M., Medina R., Gonzalez-Ondina J., Osorio, A., Mendez F.J., Garcia E. 2007. An integrated coastal modelling system for analyzing beach processes and beach restoration projects. SMC Comp. Geosci. 33 (7), 916-931.

González M., Medina R., Losada M.A. 2010. On the design of beach nourishment projects using static equilibrium concepts: application to Spanish coast. Coastal Engineering 57, 227-240 Special Issue.

Jimenez J., Sanchez-Arcilla A. 2004. A long-term (decadal scale) evolution model for microtidal barrier systems, Coastal Eng., 51, $749-764$

Khalifa M. A., El Ganainy M. A., Nasr R. I. 2009. Transformation and Longshore Sediment Transport Evaluation for the Egyptian Northern Coast via Extending Modern Formulae. Journal of Coastal Research, 25 (3), 755-767.

Martin L., Suguio K., Dominguez J.M.L, Flexor J.M. 1997. Geologia do Quaternário Costeiro do Litoral Norte do Rio de Janeiro e do Espírito Santo. CPRM (Serviço Geológico do Brasil) e FAPESP (Fundação de Amparo à Pesquisa de São Paulo). São Paulo, SP.112p.

Martin L., Suguio K. Flexor J.M., Archanjo J.D. 1996. Coastal Quaternary formations of the southern part of the State of Espírito Santo (Brazil).An. Acad. bras.Cien. 68(3):389-404.

Martin L., Suguio K., Flexor J.M. 1993. As flutuações de nível do mar durante o Quaternário superior e a evolução geológica de “deltas" brasileiros. Boletim IG-USP. Publ. Especial 15.186 p.

Mil-Homens J., Ranasinghe R., Van Thiel De Vries J.S.M., Stive M.J.F. 2013. Re-evaluation and improvement of three commonly used bulk longshore sediment transport formulas, Coastal Engineering 75, 29-39.

Oliveira F.S.B.F., Oliveira T.C.A., Silva R., Larangeiro S.H.C.D. 2004. Dinâmica Sedimentar do Trecho Litoral Praia da VieiraPraia Velha. Hidrodinâmica e Transporte Longitudinal de Sedimentos. 7W Congresso da Água, APRH, Lisbon, Portugal, p. 15

Raabe A.L.A., Klein A.H.F., Gonzalez M., Medina R., 2010. MEPBAY and SMC: software tools to support different operational levels of headland bay beaches in coastal engineering projects. Coastal Engineering 57, 213-226 Special Issue.

Reguero B.G., Menéndez M., Méndez F.Z., Mínguez R., Losada I.J. 2012. A Global Ocean Wave (GOW) calibrated reanalysis from 1948 onwards. Coastal Engineering 65, 38-55.

Silva A.N, Taborda R., Antunes C., Catalão J., Duarte J. 2013. Understanding the coastal variability at Norte beach, Portugal. Journal of Coastal Research. 65, 2173-2178.
Viola C. N. A, Grifoll M. Palalane J., Oliveira T. C. A. 2014. Sea wave propagation from offshore to Maputo's coast. Application to longshore sediment transport assessment. Water Science \& Technology, 69 (12), 2438-2445..

Recebido 05 de junho de 2014 Aceito 09 de abril de 2015 